\title{
Investigating the adsorption of 2-mercaptothiazoline on activated carbon from aqueous systems
}

\author{
Chiung-Fen Chang, ${ }^{\mathrm{a}, *}$ Ching-Yuan Chang, ${ }^{\mathrm{a}}$ and Wolfgang Höll ${ }^{\mathrm{b}}$ \\ ${ }^{a}$ Graduate Institute of Environmental Engineering, National Taiwan University, Taipei 106, Taiwan \\ ${ }^{\mathrm{b}}$ Forschungszentrum Karlsruhe, Institute for Technical Chemistry, Section WGT, P.O. Box 3640, 76021 Karlsruhe, Germany
}

Received 15 June 2003; accepted 12 September 2003

\begin{abstract}
2-Mercaptothiazoline (2-MT) is widely used as an organic corrosive as well as a diffusion inhibitor due to its high ability to form metalchelate Schiff base complexes. This study investigated the elimination of 2-MT from aqueous systems with adsorption process to reach the goal of sustainable use of water resources. The Freundlich and Langmuir adsorption isotherms were adopted to examine the adsorption behavior of two types of 2-MT (i.e., 2-MT molecule and 2-MT complex) on activated carbon (AC) Chemviron Filtrasorb 400 (F 400) in three different solutions. The results of adsorption isotherm data showed that the adsorption ability of 2-MT molecule is much higher than that of 2-MT complex, whose ability may be reduced due to the electrostatic repulsion. The good applicability of Langmuir adsorption isotherm to experimental data indicated that the adsorption of 2-MT complexes on F 400 might be limited to a monolayer. Higher ionic strength and lower $\mathrm{pH}$ value of the solutions promoted the uptake of 2-MT onto F 400 from the solutions. In addition, not only 2-MT molecule and complex but also $\mathrm{Cu}(\mathrm{II})$ can be adsorbed on the surface of $\mathrm{F} 400$, which was demonstrated by energy dispersive analysis of X-ray (EDAX). Effects of the two major interactions, chemical and physical interactions, on the adsorption of 2-MT on F 400 were compared as well in this study.
\end{abstract}

(c) 2003 Elsevier Inc. All rights reserved.

Keywords: 2-Mercaptothiazoline; Adsorption isotherms; Metal complex; Physical interaction; Chemical interaction

\section{Introduction}

2-Mercaptothiazoline (2-MT) is commonly used as an organic corrosive as well as a diffusion inhibitor due to the electron donor properties of $\mathrm{N}$ and $\mathrm{S}$ atoms, which result in adsorption/complexation behavior on the surface of the metal $[1,2]$. In order to reach the goal of a sustainable process, recycling and reusing the wastewater/process water demands immediate attention, which is a task and challenge for scientific engineers. Liquid-phase adsorption, as a separation process, is widely applied in drinking water and industrial wastewater treatments. Granular activated carbon (GAC) plays an increasing role in water/wastewater treatment because it effectively removes a wide variety of organic compounds and can be easily fitted to the treatment plants, while powder-activated carbon (PAC) is still mostly used in the drinking water treatment. For successful design and oper-

\footnotetext{
* Corresponding author.

E-mail address: cychang3@ccms.ntu.edu.tw (C.-F. Chang).
}

ation, the rate and ultimate capacity of activated carbon (AC) need to be quantified according to the conditions associated with the real treatment system [3]. Adsorption isotherm is fundamental and essential as a first step in designing the process no matter what the system is (e.g., batch or fixed-bed adsorber system). Furthermore, if the ultimate capacity is computed from the adsorption isotherm, it may be used to estimate the lowest carbon dosage for the wastewater treatment to reach economic benefit. The previous study [4] showed that it is feasible to use AC to remove polyethylene glycol on the AC from the electroplating solution, thereby achieving the benefit of recycling. Hence, the adsorption process ought to be a practical technique for eliminating the 2-MT from the aqueous systems and then to reach the goal of sustainable use of the water resource.

Numerous publications in the literature on adsorption of organics on $\mathrm{AC}$ have been available in the past (for example, Refs. [3-7]). However, the real mechanisms and phenomena on the surface of $\mathrm{AC}$ during adsorption, such as microporosity, hindered diffusion, interactions between the 
new adsorbates and ACs, effects of properties of the solution, and competition of the possible adsorbates still need to be understood. The goal of this study is to investigate the adsorption behavior of 2-MT in different aqueous systems. The adsorption capacities of 2-MT affected by the ion strength, the $\mathrm{pH}$ value, and the characteristics of 2-MT component (e.g., forming metal complex or not), were also compared and discussed. In addition, the effect of microporosity of the AC on adsorption was examined to elucidate the importance of chemical and physical interactions.

\section{Materials and methods}

\subsection{Adsorbent}

Activated carbon, Chemviron Filtrasorb 400 (F 400), with a particle size range between 12 and 40 mesh was used as the adsorbent. The mean particle size of $\mathrm{F} 400, d_{p}=1.04 \mathrm{~mm}$, was calculated from the sieve analysis of the representative samples obtained from a rotating sample-splitting device by means of the weight percentages of particles in the different sieve sizes. The physical characteristics of F 400, such as the BET specific surface area, the specific external surface area, the average true particle density, particle porosity, etc., are shown in Table 1. The pretreatment of the adsorbent comprised several steps. First, the adsorbent was washed by distilled water to remove the crushed carbon fines. Second, it was dried at $383 \mathrm{~K}$ in a vacuum oven overnight and then stored in a desiccator. Finally, it was wetted in the specific wetting solutions under vacuum, prior to both bottle-point and short fixed-bed reactor (SFB-reactor) experiments.

\subsection{Aqueous systems}

Several kinds of aqueous solvents were used to investigate the adsorption behavior of 2-MT by AC, as listed in Table 2. Two pairs of aqueous solutions (e.g., $\mathrm{S}_{\mathrm{MT}, e}$ and $\mathrm{S}_{\mathrm{MT}, 0.25}$, and $\mathrm{S}_{\mathrm{MT}, 0.25}$ and $\mathrm{S}_{\mathrm{MT}, 6}$ ) were used to investigate the effects of copper sulfate and $\mathrm{pH}$, respectively. In addition, the value of the correction factor, $f_{\beta}=2.18$, for SFB-reactor experiments were average values obtained from the adsorption of standard compounds of $p$-nitrophenol (PNP) and anthraquinone-2-sulfonate (ACS). The calculation is based on the available liquid phase diffusion coefficients $\left(D_{L}\right)$ [6]. Detail computation of $f_{\beta}$ can be referred to the study of Chang [8].

\subsection{Target adsorbate}

2-Mercaptothiazoline was reagent grade provided by Merck and used as the main target adsorbate in this study.

Table 1

Physical characteristics of Chemviron Filtrasorb 400 (F 400)

\begin{tabular}{|c|c|}
\hline Property & Value \\
\hline Mesh size & $12-40$ \\
\hline Average particle diameter, $d_{p}(\mathrm{~mm})$ & 1.044 \\
\hline Specific external surface area ${ }^{\mathrm{a}}, a_{S}\left(\mathrm{~m}^{2} / \mathrm{kg}\right)$ & 5.76 \\
\hline Average true particle density ${ }^{\mathrm{b}}, \rho_{s}\left(\mathrm{~kg} / \mathrm{m}^{3}\right)$ & 2180 \\
\hline Apparent particle density ${ }^{\mathrm{b}}, \rho_{p}\left(\mathrm{~kg} / \mathrm{m}^{3}\right)$ & 1000 \\
\hline Filter layer density ${ }^{\mathrm{c}}, \rho_{F}\left(\mathrm{~kg} / \mathrm{m}^{3}\right)$ & 530 \\
\hline Particle porosity ${ }^{\mathrm{d}}, \varepsilon_{p}(-)$ & 0.54 \\
\hline Filter voidage ${ }^{\mathrm{d}, \mathrm{e}}, \varepsilon_{F}(-)$ & 0.47 \\
\hline Langmuir specific surface area ${ }^{\mathrm{f}}\left(\mathrm{m}^{2} / \mathrm{g}\right)$ & 1363 \\
\hline Total pore volume $\mathrm{g}\left(\mathrm{cm}^{3} / \mathrm{g}\right)$ & 0.616 \\
\hline Micropore volume $\mathrm{h}^{\mathrm{h}}\left(\mathrm{cm}^{3} / \mathrm{g}\right)$ & 0.443 \\
\hline Meso- and macropore volume ${ }^{\mathrm{i}}\left(\mathrm{cm}^{3} / \mathrm{g}\right)$ & 0.173 \\
\hline Average pore diameter ${ }^{\mathrm{j}}(\AA)$ & 18 \\
\hline Average pore hydraulic radius ${ }^{\mathrm{h}}(\AA)$ & 3.6 \\
\hline
\end{tabular}

${ }^{\text {a }}$ Assumed as spherical particle and calculated using $a_{s}=6 /\left(\rho_{p} d_{p}\right)$.

b Data from the pycnometry experiments.

c In a water-filled bed.

${ }^{\mathrm{d}}$ Calculated using $\varepsilon_{p}=1-\left(\rho_{p} / \rho_{S}\right)$.

e Calculated using $\varepsilon_{F}=1-\left(\rho_{F} / \rho_{p}\right)$.

${ }^{f}$ Data from the surface area determined by $\mathrm{N}_{2}$ adsorption at $77 \mathrm{~K}$ in the volumetric equipment, ASAP 2010.

g Data from the analysis by the single point method with pore size less than $800 \AA$.

h Data from the analysis by the micropore method (MP method) with pore size between 2.4 to $17.6 \AA$.

i Data from the difference between total pore volume and micropore volume.

$\mathrm{j}$ The pore size distribution is from 17 to $3000 \AA$ A determined by the BJH integration method.

Table 2

Identification and compositions of the aqueous systems

\begin{tabular}{|c|c|c|}
\hline Solution ID & Composition & Note \\
\hline $\mathrm{S}_{e}$ & $\begin{array}{l}\text { Electroplating solution, containing concentrated sulfuric acid, } \mathrm{H}_{2} \mathrm{SO}_{4 \text { (conc) }} \text {, of } 60 \mathrm{~g} \mathrm{dm}^{-3} \text {, } \\
\mathrm{CuSO}_{4} \cdot 5 \mathrm{H}_{2} \mathrm{O} \text { of } 200 \mathrm{~g} \mathrm{dm}^{-3} \text {, and concentrated hydrochloric acid, } \mathrm{HCl}_{\text {(conc) }} \text {, of } 30 \mathrm{mgdm}^{-3} \text {. } \\
\text { The } \mathrm{pH} \text { value of } \mathrm{S}_{e} \text { is around } 0.25 \text {. }\end{array}$ & $\begin{array}{l}\text { Simulating the copper electroplat- } \\
\text { ing solution. }\end{array}$ \\
\hline $\mathrm{S}_{\mathrm{MT}, e}$ & $\begin{array}{l}\text { Electroplating solution, containing concentrated sulfuric acid, } \mathrm{H}_{2} \mathrm{SO}_{4 \text { (conc) }} \text {, of } 60 \mathrm{~g} \mathrm{dm}^{-3} \text {, } \\
\mathrm{CuSO}_{4} \cdot 5 \mathrm{H}_{2} \mathrm{O} \text { of } 200 \mathrm{~g} \mathrm{dm}^{-3} \text {, and concentrated hydrochloric acid, } \mathrm{HCl}_{(\text {conc) }} \text {, of } 30 \mathrm{mg} \mathrm{dm}^{-3} \text {. } \\
\text { The } \mathrm{pH} \text { value of } \mathrm{S}_{\mathrm{MT}, e} \text { is around } 0.25 \text {. The only organic compound in the solution } \mathrm{S}_{\mathrm{MT}, e} \text { is } \\
\text { 2-mercaptothiazoline (2-MT) at various concentrations. }\end{array}$ & $\begin{array}{l}\text { Simulating the copper electroplat- } \\
\text { ing solution with various concentra- } \\
\text { tions of 2-MT. }\end{array}$ \\
\hline $\mathrm{S}_{\mathrm{MT}, 0.25}$ & $\begin{array}{l}\text { Distilled water containing } \mathrm{HCl}_{\text {(conc) }} \text {, of } 30 \mathrm{mg} \mathrm{dm}^{-3} \text {, with the same } \mathrm{pH} \text { value as electroplating } \\
\text { solution, adjusted by } \mathrm{H}_{2} \mathrm{SO}_{4(\text { conc) }} \text {. The only organic compound in the solution } \mathrm{S}_{\mathrm{MT}, 0.25} \text { is } 2-\mathrm{MT} \\
\text { at various concentrations. }\end{array}$ & \\
\hline $\mathrm{S}_{\mathrm{MT}, 6}$ & Distilled water with dissolution of 2-MT of $0-10 \mathrm{mg} \mathrm{dm}^{-3}$. The $\mathrm{pH}$ value of $\mathrm{S}_{\mathrm{MT}, 6}$ is around 6.15 . & Simulating the general wastewater. \\
\hline
\end{tabular}


The initial concentration of 2-MT for SFB-reactor experiments was around $10 \mathrm{~g} \mathrm{~m}^{-3}$, while that for the isothermal adsorption experiments was between 0 and $50 \mathrm{~g} \mathrm{~m}^{-3}$.

\subsection{Analytical measurements}

Prior to the analysis, all the samples were filtrated through a $0.45-\mu \mathrm{m}$ membrane. Three methods and three analyzers, i.e., total organic carbon (TOC) analysis (O.I.C. M-700/Carbon Analyzer Dohrmann DC-80), UV spectrophotometry (Perkin-Elmer UV/VIS Lambda 3), and high performance liquid chromatography (HPLC, HP1090) were used to determine the concentrations of organic adsorbates in this study. The analytical conditions for detection of organic adsorbate are as follows. The wavelengths used in UV spectrophotometry for 2-MT was $270 \mathrm{~nm}$. An ODS Hyperic column and a DAD diode array detector were used in HPLC. The HPLC method for the analysis of 2-MT is so-called ionpair chromatography, which is suitable for trace-level determination [9]. The eluent for HPLC analysis was composed of $73.7 \mathrm{vol} \%$ of $5.42 \mathrm{mmol}$ tetrabutylammonium hydrogensulfate in $\mathrm{H}_{2} \mathrm{O}$ and $26.3 \mathrm{vol} \%$ of acetonitrile.

\subsection{Adsorption behavior}

Due to the restricted validity of the Freundlich isotherm, the final concentrations of adsorbates were specially selected to obtain the suitable adsorption isotherms. Various weight ratios of 2-MT to AC were prepared to get different bottlepoint data. The bottles were shaken at $298 \mathrm{~K}$ until the concentrations of filtrate did not change within a range of $\pm 3 \%$. Since it is preferable to use the weight concentration units for investigating the removal efficiency in wastewater treatments, the units of $\mathrm{g} \mathrm{m}^{-3}$ and $\mathrm{g} \mathrm{kg}^{-1}$ for the liquid and solid phases were used for all the compounds in this study.

\subsection{Energy dispersive analysis of X-ray (EDAX)}

Before the EDAX analysis, the samples were continuously and repeatedly eluted by distilled water (with conductivity of about $0.8-0.9 \mu \mho \mho / \mathrm{cm}$ ) until the conductivity of the solution was the same as that of the distilled water, which can ensure that electrolytes and adsorbates, and nonspecific compounds were no longer present in the aqueous phase, nor adsorbed on the solid surface, respectively. Thus, all the samples were dried in the oven at $373 \mathrm{~K}$ over night. The experiments were carried out by a Hitachi S-2400 scanning electronic microscope with an EDAX KEVEX Level 4. The scanning energy varied between 0 and $10.23 \mathrm{keV}$ and the elapsed time was $100 \mathrm{~s}$.

\subsection{Liquid-phase diffusion coefficient}

The short fixed bed reactor experiments were used to determine the liquid-phase diffusion coefficient, $D_{L}$. The length and diameter of the SFB-reactor were 20 and $2 \mathrm{~cm}$, respectively. The amount of adsorbent (F 400) used in this test was about $3 \mathrm{~g}$ per experiment. The temperature for the solution containing 2-MT was kept constant at $298 \mathrm{~K}$, which was carefully controlled because the effect of temperature is significant for the kinetics of adsorption [6]. To avoid air bubbles in the carbon bed, the column was packed under specific wetting solution using distilled water at the $\mathrm{pH}$ value of 0.25 . The filter velocities were well controlled at $5,8,10$, 15 , and $20 \mathrm{~m} / \mathrm{h}$, respectively. The values of $D_{L}$ in different aqueous systems were calculated by using the Gnielinski correlations [10].

\section{Results and discussion}

\subsection{Formation of complex salt}

The $\mathrm{p} K_{a}$ value of 2-MT, $11 \pm 0.1$, was determined by Mahal and Mukherjee [1]. The low $\mathrm{p} K_{a}$ value may indicate that 2-MT, with the structure shown in Fig. 1, is almost completely present in molecular form without dissociation in the three solutions applied (i.e., $\mathrm{S}_{\mathrm{MT}, e}, \mathrm{~S}_{\mathrm{MT}, 0.25}$, and $\mathrm{S}_{\mathrm{MT}, 6}$ ). Fig. 1 shows that 2-MT is an electron-pair donor as the Lewis base, and may belong to the polydentate ligand because one 2-MT melecule has the electron donor properties of not only $\mathrm{N}$-atoms but also S-atoms [11]. Various initial concentrations $\left(C_{\mathrm{MT}, e, 0}\right)$ of 2-MT in the solution of $\mathrm{S}_{\mathrm{MT}, e}$ were employed to examine the formation of metal complexes. Both time variations of 2-MT concentrations $\left(C_{\mathrm{MT}, e}\right)$ in the $\mathrm{S}_{\mathrm{MT}, e}$ solution in terms of 2-MT and TOC measured by HPLC and TOC analyzers, respectively, were studied. As shown in Fig. 2 for $C_{\mathrm{MT}} e$ determined by HPLC, the residual concentrations of 2-MT $\left(C_{\mathrm{MT}, e}^{*}=\left(C_{\mathrm{MT}, e, 0}-\right.\right.$ $\left.\left.C_{\mathrm{MT}, e}\right) / C_{\mathrm{MT}, e, 0}\right)$ strongly decreased with time for $C_{\mathrm{MT}, e, 0}$ values higher than $2 \mathrm{~g} \mathrm{~m}^{-3}$, say 10 and $100 \mathrm{~g} \mathrm{~m}^{-3}$. However, the time variations of $C_{\mathrm{MT}, e}^{*}$ in terms of TOC did not show any significant differences no matter how high the value of $C_{\mathrm{MT}, e, 0}$ was in this study. This may be because the $\mathrm{Cu}(\mathrm{II})$ in the solution $\mathrm{S}_{\mathrm{MT}, e}$ forms metal chelate Schiff base complexes with 2-MT (i.e., Lewis base, providing unshared electron pairs), which cannot be determined by HPLC analysis. In addition, an interesting phenomenon was discovered in the case of $C_{\mathrm{MT}, e, 0}=250 \mathrm{~g} \mathrm{~m}^{-3}$, where the color of the solution $\mathrm{S}_{\mathrm{MT}, e}$ (close system) was changed after two week and some powdered precipitate was formed in the solution. Because $\mathrm{Cu}$ (II) is much difficult to be further oxidized to $\mathrm{Cu}$ (III) while fairly unstable in the environment, $\mathrm{Cu}$ (II) may be reduced to $\mathrm{Cu}(\mathrm{I})$ and then form neutral, water-insoluble complexes: $[\mathrm{CuCl}]_{s}$, or $\left[\mathrm{Cu}(2-\mathrm{MT})_{n} \mathrm{Cl}\right]_{s}$ when the $\mathrm{Cl}^{-}$acts as the bridge [11]. One of the possible values of $n$ is 2 (i.e.,

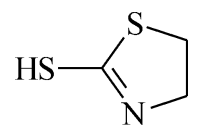

Fig. 1. Structure of 2-mercaptothiazoline. 


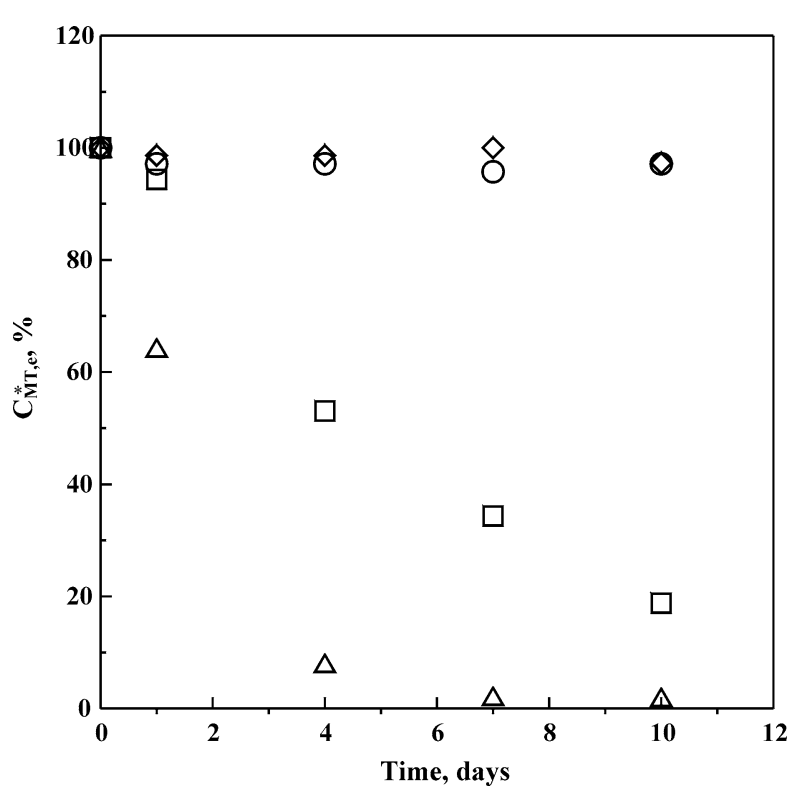

Fig. 2. Dependence of residual concentrations $\left(C_{\mathrm{MT}, e}^{*}\right)$ of 2-mercaptothiazoline (2-MT) molecules on time in the electroplating solution (Se) determined by HPLC analyzer. Experimental data with initial concentrations of 2-MT $\left(C_{\mathrm{MT}, e, 0}\right)$ for $1(\bigcirc), 2(\diamond), 10(\square)$, and $100 \mathrm{~g} \mathrm{~m}^{-3}(\triangle)$, respectively. $C_{\mathrm{MT}, e}^{*}=\left(C_{\mathrm{MT}, e, 0}-C_{\mathrm{MT}, e}\right) / C_{\mathrm{MT}, e, 0} ; C_{\mathrm{MT}, e}=$ concentration of 2-MT at time $t$.

Table 3

Ionic composition of $\mathrm{F} 400$ impregnated in various solutions measured by energy disperse analysis of X-ray (EDAX)

\begin{tabular}{lrrrrrc}
\hline Sample & \multicolumn{7}{c}{ Ionic composition (wt\%) } \\
\cline { 2 - 7 } & $\mathrm{Al}$ & $\mathrm{Si}$ & $\mathrm{Fe}$ & $\mathrm{S}$ & $\mathrm{Cl}$ & $\mathrm{Cu}$ \\
\hline Original $^{\mathrm{a}}$ & 17.05 & 35.87 & 8.47 & 36.67 & 1.93 & - \\
$\mathrm{A}^{\mathrm{b}}$ & 7.08 & 18.10 & 6.04 & 41.44 & 15.29 & 11.33 \\
$\mathrm{~B}^{\mathrm{c}}$ & 9.31 & 16.23 & 3.82 & 39.13 & 14.74 & 16.76 \\
\hline
\end{tabular}

${ }^{\text {a }} \mathrm{F} 400$ activated carbon analyzed by EDAX without any pretreatment.

${ }^{\mathrm{b}} \mathrm{F} 400$ impregnated in the solution $\mathrm{S}_{e}$ with the ratio of $0.5 \mathrm{~g} / \mathrm{dm}^{3}$ for 7 days.

${ }^{c}$ F 400 impregnated in the solution $\mathrm{S}_{\mathrm{MT}, e}$ with the ratio of $0.5 \mathrm{~g} / \mathrm{dm}^{3}$ for 7 days, in which the concentrations of 2-MT is $50 \mathrm{~g} \mathrm{~m}^{-3}$.

$\left.\left[\mathrm{Cu}(2-\mathrm{MT})_{2} \mathrm{Cl}\right]\right)$. According to a previous study [12], $\mathrm{Cu}(\mathrm{I})$ may be coordinated by $2 \mathrm{~S}$ atoms, which are in the position of SH of two 2-MT molecules as illustrated in Fig. 1, and a $\mathrm{Cl}$ atom in the geometry of trigonal plane. Furthermore, between $\mathrm{Cl}$ and two 2-MT molecules, two hydrogen bonds are held in the intermolecule. The clear and exact mechanism related to electron transfer reactions would need further examination using a precise and delicate design.

\subsection{Adsorption of copper ions}

The ionic composition and EDAX spectra of F 400 samples are listed in Table 3. The result for original activated carbon $\mathrm{F} 400$ shows that the major bulk components are $\mathrm{Al}$, $\mathrm{Si}, \mathrm{Fe}, \mathrm{S}$, and $\mathrm{Cl}$, with $\mathrm{Si}$ being the most abounding element. The F 400 impregnated in $\mathrm{S}_{e}$ solution revealed that $\mathrm{Cu}(\mathrm{II})$ was indeed adsorbed by F 400 in the solution of $\mathrm{pH} 0.25$.
This may be because $\mathrm{Cu}$ (II) forms a coordination complex with the functional group on the F 400 surface so that the $\mathrm{Cu}$ (II) was not washed out with the distilled water. The previous study [5] showed that when the $\mathrm{pH}$ value of the solution was lower than 3 , the amount of $\mathrm{Cu}$ (II) adsorbed from aqueous solution was very high and behaved like the type I phenomenon of the BET classification, which can be interpreted as the adsorption of $\mathrm{Cu}$ (II) on $\mathrm{F} 400$ being limited to a monolayer. Similar results were obtained when the F 400 was impregnated in $\mathrm{S}_{\mathrm{MT}, e}$ solution. For the latter two cases with $\mathrm{S}_{e}$ and $\mathrm{S}_{\mathrm{MT}, e}$, the weight percentages of both $\mathrm{S}$ and $\mathrm{Cl}$ on the sample surface increased, leading to the interpretation that the sulfate and $\mathrm{Cl}$ may also be adsorbed on $\mathrm{F} 400$ due to the electrical neutralization in $\mathrm{S}_{e}$ and $\mathrm{S}_{\mathrm{MT}, e}$ solutions. However, in solution $\mathrm{S}_{\mathrm{MT}, e}$, additional phenomena may occur, i.e., the adsorption of 2-MT and $\mathrm{Cu}$ (II) complex salt.

\subsection{Adsorption behavior}

For investigation of the adsorption behavior of 2-MT on F 400 in this study, the initial concentrations of 2-MT were smaller than $60 \mathrm{~g} \mathrm{~m}^{-3}$ in order to ensure that no precipitation occurred in the aqueous system before the adsorption equilibrium was reached. The empirical Freundlich and Langmuir isotherms, which correspond to the heterogeneous and homogeneous adsorbent surfaces, respectively, were used to correlate the experimental data as follows:

$\begin{aligned} q_{e} & =k_{F} C_{e}^{1 / n_{F}}, \\ q_{e} & =\frac{Q_{L} K_{L} C_{e}}{1+K_{L} C_{e}} .\end{aligned}$

In Eqs. (1) and (2), $q_{e}$ and $C_{e}$ are the adsorbate concentrations in the solid and liquid phases at equilibrium, respectively. The $k_{F}$ and $n_{F}$ are the Freundlich isotherm constants, which represent the adsorption capacity and strength of adsorption, respectively. $Q_{L}$ and $K_{L}$ are the Langmuir isotherm constants, in which the first one represents the monolayer adsorption capacity. The constants in the models can be obtained by linearizing the above equations as follows:

$\ln q_{e}=\ln k_{F}+n_{F} \ln C_{e}$,
$\frac{1}{q_{e}}=\frac{1}{Q_{L} K_{L}} \frac{1}{C_{e}}+\frac{1}{Q_{L}}$.

The results are shown in Table 4 and Fig. 3.

As mentioned above, there are two types of adsorbates (i.e., 2-MT molecule and 2-MT complex) in the $\mathrm{S}_{\mathrm{MT}, e}$ solution, while 2-MT molecules only exist in the solutions

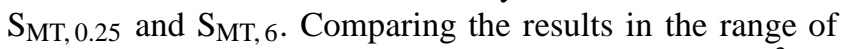
equilibrium concentrations $\left(C_{e}\right)$ between 0 and $2 \mathrm{~g} \mathrm{~m}^{-3}$ indicates that the adsorption capacity (expressed by $k_{F}$ ) of 2-MT on $\mathrm{F} 400$ in $\mathrm{S}_{\mathrm{MT}, e}$ was greater than those in $\mathrm{S}_{\mathrm{MT}, 0.25}$ and $\mathrm{S}_{\mathrm{MT}, 6}$, but the reverse held for the strength of adsorption of $n_{F}$ of 2-MT on F 400. The higher $k_{F}$ values may be because the double layer of activated carbon F 400 was 
very effectively reduced when copper sulfate is present in $\mathrm{S}_{\mathrm{MT}, e}$, while the lower $n_{F}$ may be attributed to the reason that the unpaired ions of 2-MT are also compressed resulting in low strength of adsorption. However, the experimental data show that there is not much difference of $k_{F}$ between solutions of $\mathrm{S}_{\mathrm{MT}, e}$ and $\mathrm{S}_{\mathrm{MT}, 0.25}$ compared to the solution $\mathrm{S}_{\mathrm{MT}, 6}$, in which the $k_{F}$ is much lower than those in $\mathrm{S}_{\mathrm{MT}, e}$ and $\mathrm{S}_{\mathrm{MT}, 0.25}$. It is obvious that $k_{F}$ for the adsorption of 2-MT molecules on F 400 is much greater than for 2-MT complex no matter what kind of solution systems contains the 2-MT molecules. Comparing the Freundlich isotherm constants of 2-MT molecules (with $C_{e}$ of $0-2 \mathrm{~g} \mathrm{~m}^{-3}$ ) and complexes (with $C_{e}$ of $0-40 \mathrm{~g} \mathrm{~m}^{-3}$ ) in $\mathrm{S}_{\mathrm{MT}, e}$, the close $n_{F}$ but very different $k_{F}$ values show that the adsorption strength of 2-MT (molecules and complexes) on F 400 are similar. This may be due to the fact that the adsorption of 2-MT

\section{Table 4}

Isotherm parameters and correlation coefficients $\left(r^{2}\right)$ of 2-MT in various aqueous systems

\begin{tabular}{lccc}
\hline & \multicolumn{3}{c}{ Equilibrium concentration range: $0-2 \mathrm{~g} \mathrm{~m}^{-3}$} \\
System & $k_{F}\left(\mathrm{~g}_{F}^{(1-1 / n)} \mathrm{kg}^{-1} \mathrm{~m}_{F}^{3 / n}\right)$ & $n_{F}(-)$ & $r_{F}^{2}$ \\
\hline $\mathrm{S}_{\mathrm{MT}, e}$ & 230 & 1.5 & 0.9759 \\
$\mathrm{~S}_{\mathrm{MT}, 0.25}$ & 202 & 3.3 & 0.9686 \\
$\mathrm{~S}_{\mathrm{MT}, 6}$ & 84 & 2.1 & 0.9054 \\
& \multicolumn{2}{c}{ Equilibrium concentration range: $0-40 \mathrm{~g} \mathrm{~m}^{-3}$} & \\
System & $k_{F}\left(\mathrm{~g}_{F}^{(1-1 / n)} \mathrm{kg}^{-1} \mathrm{~m}_{F}^{3 / n}\right)$ & $n_{F}(-)$ & $r_{F}^{2}$ \\
\hline $\mathrm{S}_{\mathrm{MT}, e}$ & 18 & 1.58 & 0.9451 \\
& $Q_{L}\left(\mathrm{~g} \mathrm{~kg}^{-1}\right)$ & $K_{L}\left(\mathrm{~m}^{3} \mathrm{~g}^{-1}\right)$ & $r_{L}^{2}$ \\
\cline { 2 - 4 } & 270 & 0.043 & 0.9791 \\
\hline
\end{tabular}

molecules and complexes on $\mathrm{F} 400$ surface is resulting from the hydrophobic attraction while the electrostatic repulsion reduces the adsorption capacity of 2-MT complex on the surface. Furthermore, the $\mathrm{Cu}(\mathrm{II})$ species adsorbed on the F 400 surface also have the same negative effect on 2-MT complexes. Therefore, the adsorption of 2-MT complexes on F 400 is not favorable in $\mathrm{S}_{\mathrm{MT}, e}$ solution.

Adsorption of organic matters onto activated carbon normally occurs because of two major interactions: physical and chemical interactions, from which the former mainly refers to the size exclusion and microporosity effect and the latter chiefly concerns about the chemical nature of the adsorbent and adsorbate, and the composition of the solvent [7]. From the results of the SFB-reactor experiments, the liquid-phase diffusion coefficients $\left(D_{L}\right)$ of 2-MT molecule in three solutions were $5.3 \times 10^{-10} \mathrm{~m}^{2} / \mathrm{s}$ in $\mathrm{S}_{\mathrm{MT}, 6}, 8.0 \times 10^{-10} \mathrm{~m}^{2} / \mathrm{s}$ in $\mathrm{S}_{\mathrm{MT}, 0.25}$, and $9.7 \times 10^{-10} \mathrm{~m}^{2} / \mathrm{s}$ in $\mathrm{S}_{\mathrm{MT}, e}$, respectively, in which the first was calculated using Wilke-Chang correlations [13]. Furthermore, if the Stokes-Einstein equation $\left(D_{L}=(k T) / 6 \pi \mu_{B} R\right)$ is adopted to calculate the hydrodynamic diameter $(R)$ of 2-MT in different solutions, the values of $R$ in sequence are 2.8, 5.2, and $8.2 \AA$ for the solutions $\mathrm{S}_{\mathrm{MT}, e}, \mathrm{~S}_{\mathrm{MT}, 0.25}, \mathrm{~S}_{\mathrm{MT}, 6}$, respectively. It was expected that the high adsorption potential of 2-MT of low molecular weight on F 400 can be obtained due to the high microporosity of the $\mathrm{F} 400$. Comparing the ratios of the average diameter of the pore size (18 $\AA$ ) to the hydrodynamic diameters $(2.8,5.2$, and $8.2 \AA)$ of the 2-MT in various solutions, it can be noted that at low concentrations 2-MT can access all the micropores of the F 400 in all three solutions. Thus, the adsorption of 2-MT at low concentrations does not result

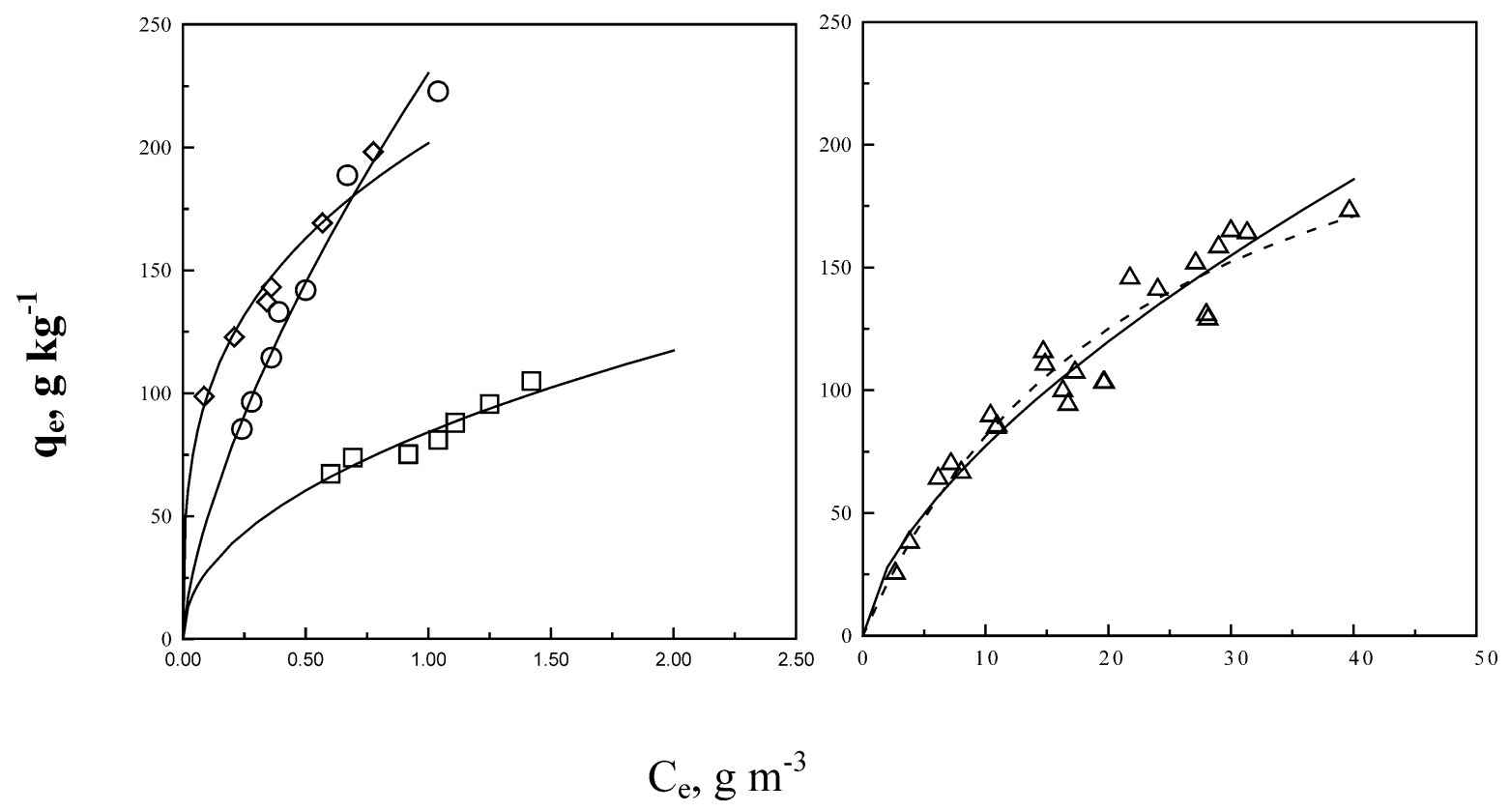

Fig. 3. Adsorption isotherms of 2-MT molecule and complex in various solutions. Experimental data of 2-MT species in solutions of $\mathrm{S}_{\mathrm{MT}, e}(\mathrm{O}), \mathrm{S}_{\mathrm{MT}, 0.25}(\square)$, and $\mathrm{S}_{\mathrm{MT}, 6}(\diamond)$ with the equilibrium concentrations of 2-MT molecules in the range 0-2 $\mathrm{g} \mathrm{m}^{-3}$. ( $\triangle$ ) Experimental data of 2-MT copper complex in $\mathrm{S}_{\mathrm{MT}, e}$ solution with equilibrium concentrations of 2-MT complex species in the range of $0-40 \mathrm{~g} \mathrm{~m}^{-3}$. (-), (---) data simulated by Freundlich and Langmuir isotherms (values of $r^{2}$ are indicated in Table 3). 
in a steric hindrance inside the micropores, and, therefore, the size exclusion and microporosity effect may not affect the adsorption of 2-MT molecules inside the micropores of the F 400. However, the uptake of 2-MT molecules on F 400 from the solution $\mathrm{S}_{\mathrm{MT}, 6}$ was indeed reduced very strongly. A further comparison of the uptake of 2-MT molecules in $\mathrm{S}_{\mathrm{MT}, e}$ and $\mathrm{S}_{\mathrm{MT}, 0.25}$ with $\mathrm{S}_{\mathrm{MT}, 6}$ indicates that lower $\mathrm{pH}$ value and higher ionic strength promote the uptake of 2-MT molecules on F 400. However, the addition of more copper sulfate (i.e., increase of the ionic strength) could not significantly enhance the adsorption of 2-MT molecules on F 400 when the $\mathrm{pH}$ of solution had the low value of 0.25 .

When the 2-MT molecule formed metal-chelate Schiff base complexes (2-MT complex) in the $\mathrm{S}_{\mathrm{MT}, e}$, the adsorption phenomena could be considered as an organic ion adsorbed on the surfaces of F 400. The F 400 surface acquired a positive surface charge by the adsorption of the ions onto the surface, due to the cause that the $\mathrm{pH}$ value of the solution is lower than $\mathrm{pH}_{\mathrm{PZC}}$. Organic ions are electrostatically repelled from the diffusion layer so that their concentration in the diffusion layer is lower than that in the bulk solution. An increase of the ionic strength of the bulk solution by the electrolyte not only increases the adsorption of organic ions (i.e., the surface excess) but also compresses the diffusion part of double layer [14]. The forces responsible for the adsorption of the organic ions may mainly be due to the noncoulombic force, such as dispersion forces, hydrogen bonding, and so on in $\mathrm{S}_{\mathrm{MT}, e}$ [14]. The ion exchange and surface complexation formed by the 2-MT complex and the functional groups play minor roles in the adsorption of 2-MT complex on the mainly hydrophobic surface of the F 400. In addition, the uptake of 2-MT complexes on F 400 is smaller in $\mathrm{S}_{\mathrm{MT}, e}$, due to (1) the electrostatic repulsion between the adsorbed 2-MT complexes, (2) the occupation of the surface by $\mathrm{Cu}(\mathrm{II})$, and (3) the occurrence of a positively charged surface when the $\mathrm{pH}$ of the solution was lower than $\mathrm{pH}$ PZC. Therefore, the adsorption of 2-MT complex might be limited to the monolayer on the positively charged surface of F 400 . It is noted that the Freundlich isotherm can often describe the experimental data satisfactorily in a small concentration range. However, when the Langmuir isotherm well describes the experimental results by comparing the values of the correlation coefficients, this implies that the adsorption of the adsorbate on the adsorbent is likely limited to the monolayer. As a result, it is concluded that the adsorption of 2-MT complex onto the positively charged surface of $F 400$ might be limited to a monolayer.

\section{Conclusions}

The formation of metal-chelate Schiff base complexes (i.e., 2-mercaptothiazoline copper complex, 2-MT complex) in the electroplating solution $\left(\mathrm{S}_{e}\right)$ has been demonstrated by analyzing the samples with HPLC and TOC analyzers when the initial concentration of 2-MT in $\mathrm{S}_{e}$ was greater than $2 \mathrm{~g} \mathrm{~m}^{-3}$. Therefore, two types of adsorbates (i.e., 2-MT molecule and 2-MT complex) have to be assumed in the solution $\mathrm{S}_{\mathrm{MT}, e}$, while only 2-MT molecules exist in the solutions $\mathrm{S}_{\mathrm{MT}, 0.25}$ and $\mathrm{S}_{\mathrm{MT}, 6}$. In addition, electron transfer reactions may occur in the system to reduce $\mathrm{Cu}(\mathrm{II})$ to $\mathrm{Cu}(\mathrm{I})$ and then form neutral, water-insoluble complexes. The EDAX spectra of activated carbon $\mathrm{F} 400$ show that the major bulk components are $\mathrm{Al}, \mathrm{Si}, \mathrm{Fe}, \mathrm{S}$, and $\mathrm{Cl}$, with $\mathrm{Si}$ being the most abounding element. In addition, $\mathrm{Cu}$ (II) can be adsorbed onto $\mathrm{F} 400$ in the solution $\mathrm{S}_{e}$ at $\mathrm{pH} 0.25$ due to the formation of a coordinate complex with the functional group on the F 400 surface.

Adsorption of 2-MT on F 400 demonstrates that the adsorption capacity for 2-MT molecules in sequence is $\mathrm{S}_{\mathrm{MT}, e}=\mathrm{S}_{\mathrm{MT}, 0.25}>\mathrm{S}_{\mathrm{MT}, 6}$ in the range of equilibrium concentrations between 0 and $2 \mathrm{~g} \mathrm{~m}^{-3}$. The 2-MT complexes exhibit the smallest uptake by $\mathrm{F} 400$. The excellent applicability of the Langmuir isotherm to experimental data indicate that the adsorption of 2-MT complexes on F 400 might be limited to a monolayer. The adsorption strength of 2-MT molecules and complexes on F 400 result from their similar hydrophobic attraction. The adsorption capacity of the former was much greater than that of the latter due to the electrostatic repulsion of 2-MT complexes on the surface which inhibit the adsorption of 2-MT complexes on F 400 in a $\mathrm{S}_{\mathrm{MT}, e}$ solution.

The hydrodynamic diameters $(R)$ of 2-MT in various solutions calculated by Stokes-Einstein equation are 2.8, 5.2, and $8.2 \AA$ for the solutions $\mathrm{S}_{\mathrm{MT}, e}, \mathrm{~S}_{\mathrm{MT}, 0.25}, \mathrm{~S}_{\mathrm{MT}, 6}$, respectively. A high adsorption capacity of 2-MT of low molecular weight onto F 400 can be obtained due to high microporosity and nonsteric hindrance, so that physical interaction does not play an important role in adsorption of 2-MT molecules on F 400 in this study. Lower $\mathrm{pH}$ value and higher ionic strength promote the uptake of 2-MT molecules on F 400, while the uptake of 2-MT complexes on F 400 was restrained by electrostatic repulsion. Thus, the chemical interactions are significant and predominant over the physical interactions with respect to the adsorption capacity of 2-MT molecules on F 400.

\section{Acknowledgment}

The authors thank the Powder Technology Laboratory of the Chemical Engineering Department of the National Taiwan University for assistance in powder characterization.

\section{Appendix A. Nomenclature}

AC Activated carbon

ACS Anthraquinone-2-sulfonate

$a_{s} \quad$ Specific external surface area $\left(\mathrm{m}^{2} / \mathrm{kg}\right)$

BET The specific surface area obtained following the Brunauer, Emmett, and Teller theory 
$C_{e} \quad$ Adsorbate concentration in the liquid phase at equilibrium with $q_{e}\left(\mathrm{~g} \mathrm{~m}^{-3}\right)$

$C_{\mathrm{MT}} \quad$ Concentration of 2-MT

$C_{\mathrm{MT}, e} \quad C_{\mathrm{MT}}$ in $\mathrm{S}_{\mathrm{MT}, e}$ solution at time $t$

$C_{\mathrm{MT}, e, 0} \quad C_{\mathrm{MT}, e}$ at time $=0$

$C_{\mathrm{MT}, e}^{*} \quad\left(C_{\mathrm{MT}, e, 0}-C_{\mathrm{MT}, e}\right) / C_{\mathrm{MT}, e, 0}$

$D_{L} \quad$ Liquid diffusivity, liquid diffusion coefficient $\left(\mathrm{m}^{2} / \mathrm{s}\right)$

$d_{p} \quad$ Mean particle size $(\mathrm{mm})$

EDAX Energy dispersive analysis of $\mathrm{X}$-rays

F 400 Chemviron Filtrasorb 400

$f_{\beta} \quad$ Correction factor for particle shape (ratio of experimental to correlation values of $\beta_{L} a_{s}=\left(\beta_{L} a_{s}\right)_{\exp } /$ $\left.\left(\beta_{L} a_{s}\right)_{\text {cor }}\right)$

GAC Granular activated carbon

$k \quad$ Boltzmann constant, $1.381 \times 10^{-23} \mathrm{~J} \mathrm{~K}^{-1}$

$k_{F} \quad$ Freundlich isotherm constant as specified in Eq. (1)

$K_{L} \quad$ Langmuir isotherm constant as specified in Eq. (2)

MP Micropore

2-MT 2-mercaptothiazoline

$n_{F} \quad$ Freundlich isotherm constant as specified in Eq. (1)

PAC Powder activated carbon

PNP $p$-Nitrophenol

$\mathrm{pH}_{\mathrm{PZC}} \mathrm{pH}$ value at the point of zero charge

$q_{e} \quad$ Adsorbate concentration in solid phase at equilibrium with $C_{e}(\mathrm{mg} / \mathrm{g}$ or $\mathrm{mol} / \mathrm{kg})$

$Q_{L} \quad$ The monolayer adsorption capacity as specified in Eq. (2)

$R \quad$ Solute radius, hydrodynamic radius, equivalent radius $(\AA)$

$r^{2} \quad$ Correlation coefficient

$\mathrm{S}_{e} \quad$ Electroplating solution

$\mathrm{S}_{\mathrm{MT}, e}$, Aqueous solutions with different compositions as

$\mathrm{S}_{\mathrm{MT}, 0.25}$, specified in Table 2

$\mathrm{S}_{\mathrm{MT}, 6}$

SFB Short fixed-bed

$T \quad$ Absolute temperature (K)

TOC Total organic carbon

$t \quad$ Adsorption time or elapsed time (h)

ZPC Zero point charge
$\beta_{L} \quad$ Film mass transfer coefficient gained from SFB reactor $(\mathrm{m} / \mathrm{s})$

$\beta_{L} a_{s} \quad$ Specific value of $\beta_{L}\left(\mathrm{~m}^{3} / \mathrm{s} / \mathrm{kg}\right)$

$\left(\beta_{L} a_{s}\right)_{\exp }, \quad \beta_{L} a_{s}$ obtained by experiments and correlation

$\left(\beta_{L} a_{s}\right)_{\text {cor }}$

$\varepsilon_{p} \quad$ Adsorbent porosity

$\varepsilon_{F} \quad$ Filter bed voidage

$\mu \quad$ Dynamic viscosity (cp)

$\mu_{B} \quad \mu$ of solvent (cp)

$\mu_{w} \quad \mu$ of water (cp)

$v \quad$ Kinetic viscosity $\left(\mu / \rho, \mathrm{m}^{2} / \mathrm{s}\right)$

$\rho \quad$ Density $\left(\mathrm{kg} / \mathrm{m}^{3}\right)$

$\rho_{F} \quad$ Filter bed layer density $\left(\mathrm{kg} / \mathrm{m}^{3}\right)$

$\rho_{p} \quad$ Apparent particle density $\left(\mathrm{kg} / \mathrm{m}^{3}\right)$

$\rho_{s} \quad$ Average true particle density $\left(\mathrm{kg} / \mathrm{m}^{3}\right)$

\section{References}

[1] H.S. Mahal, T. Mukherjee, Radiat. Phys. Chem. 54 (1999) 29.

[2] C.L. Fang, General Concepts of Additives in the Electroplating Solution, Finishing Science Publication, Taipei, 1996.

[3] H. Sontheimer, J.C. Crittenden, R.S. Summers, Activated Carbon for Water Treatment, DVGW-Forschungsstelle, Karlsruhe, 1998.

[4] C.F. Chang, C.Y. Chang, W.T. Tsai, J. Colloid Interface Sci. 232 (2000) 207.

[5] R.C. Bansal, J.B. Donnet, F. Stoeckli, Active Carbon, Dekker, New York, 1988.

[6] R. Isabel, Temperature Dependence of the Kinetics of Activated Carbon Adsorption, internship work performed at Heinrich-SontheimerLaboratory, DVGW-Technologiezentrum Wasser, Karlsruhe, 1998.

[7] T. Karanfil, J.E. Kilduff, Environ. Sci. Technol. 33 (1999) 3217.

[8] C.F. Chang, A Study on the application of activated carbon for the removal of organic additives from simulated printed wiring electroplating solution, Ph.D. dissertation, Graduate Institute of Environmental Engineering, National Taiwan University, Taipei, 2002.

[9] F.T. Lange, M. Wenz, H.-J. Brauch, J. High Resolution Chromatogr. 18 (1995) 243.

[10] V. Gnielinski, Verfahrenstechnik 12 (6) (1978) 363.

[11] A.C. Cotton, G. Wilkinson, C.A. Murillo, M. Bochmann, Advanced Inorganic Chemistry, 6th ed., Wiley, New York, 1999.

[12] M. Maekawa, S. Kitagawa, Y. Nozaka, M. Munakata, T. Kurodasowa, Anal. Sci. 9 (6) (1993) 887-888.

[13] C.R. Wilke, P. Chang, Am. Inst. Chem. Eng. J. 1 (2) (1955) 264.

[14] F.F. Cantwell, Ion Exch. Sol. Extract. 9 (1985) 339. 